\title{
Physical activity during video capsule endoscopy correlates with shorter bowel transit time
}

\section{(c) $(1) \ominus$}

Authors

Peter P. Stanich ${ }^{1}$, Joshua Peck ${ }^{1}$, Christopher Murphy ${ }^{1}$, Kyle M. Porter ${ }^{2}$, Marty M. Meyer ${ }^{1}$

Institutions

1 Section of Capsule Endoscopy, Division of Gastroenterology, Hepatology and Nutrition, The Ohio State University Wexner Medical Center, Columbus, Ohio, United States

2 Center for Biostatistics, The Ohio State University Wexner Medical Center, Columbus, Ohio, United States

submitted 20.2.2017

accepted after revision 21.6.2017

\author{
Bibliography \\ DOI https://doi.org/10.1055/s-0043-115385 | \\ Endoscopy International Open 2017; 05: E856-E860 \\ (c) Georg Thieme Verlag KG Stuttgart · New York \\ ISSN 2364-3722
}

Corresponding author

Marty M. Meyer, MD, Ohio State University Wexner Medical Center, 395 West 12th Avenue, Suite 200, Columbus,

$\mathrm{OH} 43210$, United States

Fax: +1-614-293-8518

Marty.Meyer@OSUMC.edu

\section{ABSTRACT}

Background and study aim Video capsule endoscopy (VCE) is limited by reliance on bowel motility for propulsion, and lack of physical activity has been proposed as a cause of incomplete studies. Our aim was to prospectively investi- gate the association between physical activity and VCE bowel transit.

Patients and methods Ambulatory outpatients receiving VCE were eligible for the study. A pedometer was attached at the time of VCE ingestion and step count was recorded at the end of the procedure. VCE completion was assessed by logistic regression models, which included step count (500 steps as one unit). Total transit time was analyzed by Cox proportional hazards models. The hazard ratios (HR) with $95 \%$ confidence interval $(\mathrm{Cl})$ indicated the "hazard" of completion, such that HRs $>1$ indicated a reduced transit time. Results A total of 100 patients were included. VCE was completed in 93 patients (93\%). The median step count was 2782 steps. Step count was not significantly associated with VCE completion (odds ratio 1.45, $95 \% \mathrm{Cl} 0.84,2.49$ ). Pedometer step count was significantly associated with shorter total, gastric, and small-bowel transit times (HR $1.09,95 \% \mathrm{Cl} 1.03,1.16$; HR 1.05, $95 \% \mathrm{Cl} 1.00,1.11$; HR $1.07,95 \% \mathrm{Cl} 1.01,1.14$, respectively). Higher body mass index (BMI) was significantly associated with VCE completion (HR 1.87, $95 \% \mathrm{Cl} 1.18,2.97)$ and shorter bowel transit times (HR 1.05, $95 \% \mathrm{Cl} 1.02,1.08$ ).

Conclusions Increased physical activity during outpatient VCE was associated with shorter bowel transit times but not with study completion. In addition, BMI was a previously unreported clinical characteristic associated with VCE completion and should be included as a variable of interest in future studies.

\section{Introduction}

Video capsule endoscopy (VCE) is a noninvasive, wireless modality that has been increasingly utilized to examine the smallbowel mucosa since its introduction in 2000 [1]. A completed VCE depends upon natural gastrointestinal motility to propel the device through the esophagus, stomach, small intestine, and into the colon during the battery lifespan. Systematic review of published literature has revealed a completion rate of only $83.5 \%$ for the standard 8-hour device [2]. Incomplete studies are unfortunate and may result in missed distal lesions in the unexamined small bowel, as well as the need for repeat procedures.

Inpatient status has consistently been identified as a risk factor for incomplete VCE and this is often postulated to be secondary to decreased mobility of hospitalized patients [3-5]. There is a solid physiological rationale to support this, as physical activity has been shown to promote accelerated intestinal transit of gas and liquids [6, 7]. To further support this hypothesis, a study by Shibuya et al. [8] retrospectively and indirectly assessed activity levels of patients undergoing VCE. Although crudely measured, decreased activity was a significant risk factor for incomplete VCE after multivariate analysis $(P=0.048)$. To 
date, there have been no prospective studies to further clarify this.

The primary aim of our study was to prospectively investigate the association between physical activity and VCE completion. An objective measure of physical activity was quantified through pedometer step count reading, which has been validated as a measure of physical activity in clinical research and is the preferred measuring device $[9,10]$. We also correlated physical activity levels with gastric, small-bowel, and total bowel transit times as secondary outcome measures.

\section{Patients and methods}

\section{Patients}

This study was approved by the appropriate Institutional Review Board prior to initiation. All patients undergoing outpatient VCE underwent an initial assessment for inclusion in the study. Inclusion criteria included age 18 years or older, capacity to provide consent for both the VCE and the study, and being ambulatory (i.e. not a wheelchair user). Exclusion criteria included: the need for a patency capsule as pre-determined by the referring physician owing to risk factors for capsule retention; endoscopic delivery of the VCE; previous gastric or smallbowel surgery; gastroparesis and/or use of prokinetics such as metoclopramide or azithromycin; a history of previous VCE retained in the stomach for the duration of the battery life; a history of small-bowel resection; inpatient status.

After study consent, data were collected regarding patient sex, age, body mass index (BMI), indication for procedure, bowel preparation use prior to capsule, medications, and clinical history of diabetes, thyroid disease or inflammatory bowel disease. Immediately after VCE ingestion, a Yamax Digi-Walker SW-701 (Yamax Corp., Tokyo, Japan) was attached to the patient at the belt or waistband in the mid-line of the thigh according to manufacturer recommendations. This is a commercially available pedometer that has been validated in multiple peer-reviewed studies as an accurate research tool $[11,12]$. The face of the pedometer was then sealed with a sticker for 8 hours to avoid the theoretical concern of visible step-count readings altering the typical activity levels of patients. At the conclusion of the VCE study, the study staff unsealed the device and the step-count reading was recorded.

VCE completion, gastric transit time, small-bowel transit time, total transit time, diagnostic findings, and bowel preparation rating (preparation was considered adequate if greater than $75 \%$ of the luminal segments were visible [13]) were obtained from the standard VCE report performed by a single physician (M.M.M.), who was blinded to study participation and data at the time.

\section{Capsule endoscopy}

All VCEs were performed with the PillCam SB2 Small-Bowel Video Capsule (Given Imaging, Yoqneam, Israel) with the standard 8-hour battery lifespan and interpreted using the associated software. Our standard outpatient capsule protocol was performed for all patients. This included instructions to avoid sucralfate or iron for 5 days prior to the procedure, clear liquid diet starting at 10:00 the day before the procedure, $2 \mathrm{~L}$ of a polyethylene glycol 3350, sodium bicarbonate, sodium chloride, and potassium chloride bowel purgative solution (Trilyte; Alaven Pharmaceutical LLC, Marietta, Georgia, USA) at 16:30 the day before the procedure, and no further oral intake from midnight before the day of the procedure. Patients were instructed that clear liquid intake could be started at 2 hours after VCE ingestion and a light meal after 4 hours.

\section{Statistical analysis}

Baseline characteristics are summarized as mean (SD) or count and percent. VCE completion was analyzed by logistic regression. Univariable models were fit using pedometer step count (500 steps as one unit), age, sex, BMI, Type 2 diabetes mellitus, inflammatory bowel disease, hypothyroidism, hyperthyroidism, iron deficiency anemia as the indication for the procedure, normal findings on the examination, and use of narcotics, anticholinergics, beta-blockers, calcium-channel blockers or thyroid medication as independent variables. Pedometer step count and variables with $P$ values $\leq 0.10$ were included in a multivariable model, with the exception of normal vs. non-normal findings, which had a very wide confidence interval for the odds ratio, indicating instability and imprecision. Linearity of continuous variables was assessed by loess plots and logistic regression on the quartile indicators. Odds ratios (ORs) estimated by the model are presented along with the $95 \%$ confidence intervals (Cls).

Total transit time was analyzed by Cox proportional hazards models, with patients who did not achieve VCE completion censored at 480 minutes (the length of capsule battery life). The hazard ratios (HRs) estimated by the model indicate the "hazard" of completion, such that HRs $>1$ indicate reduced transit time. They are presented along with the $95 \% \mathrm{Cls}$. Univariable models were fit with the same variables as above, and variables with $P$ values $\leq 0.10$ were included in a multivariable model. Gastric transit time and small-bowel transit time were also analyzed by multivariable Cox proportional hazards models. The gastric transit time for one patient was censored at $480 \mathrm{~min}$ utes. The small-bowel transit times for patients not achieving VCE completion were censored at 480 minutes minus the gastric transit time.

\section{Results}

A total of 105 patients met the inclusion criteria and consented to the study. Five patients were subsequently excluded from the analysis: two patients were excluded because the pedometer was not returned, two because there was a pedometer dysfunction, and one patient did not follow the procedure protocol. Baseline clinical characteristics of the patients are detailed in $>$ Table 1. Of note, 3 patients ( $3 \%$ ) were underweight (BMI<18.5), 12 patients (12\%) had normal weight (BMI 18.524.9), 34 patients (34\%) were overweight (BMI $25-29.9$ ), and 51 patients $(51 \%)$ were obese (BMI>30). The VCE was completed during the battery lifespan in 93 patients (93\%). There was gastric retention during the battery lifespan in one patient (1\%). 
- Table 1 Patient clinical characteristics.

\begin{tabular}{|c|c|}
\hline & $n=100$ \\
\hline Age, mean (SD), years & $50.9(12.3)$ \\
\hline Female sex, n (\%) & $64(64)$ \\
\hline BMI, mean (SD), kg/m² & $31.3(6.8)$ \\
\hline - Underweight (BMI<18.5), n (\%) & $3(3)$ \\
\hline - Normal (BMI 18.5-24.9), n (\%) & $12(12)$ \\
\hline - Overweight (BMI 25 - 29.9), n (\%) & $34(34)$ \\
\hline - Obese Class I (BMI 30 - 34.9), n (\%) & $17(17)$ \\
\hline - Obese Class II (BMI 35 - 39.9), n (\%) & $23(23)$ \\
\hline - Extreme obesity (BMI>40), n (\%) & $11(11)$ \\
\hline Diabetes mellitus, n (\%) & $21(21)$ \\
\hline - Type 1 & $0(0)$ \\
\hline - Type 2 & $21(21)$ \\
\hline Inflammatory bowel disease, n (\%) & $9(9)$ \\
\hline - Crohn's disease & $8(8)$ \\
\hline - Ulcerative colitis & $1(1)$ \\
\hline Thyroid disease, n (\%) & $21(21)$ \\
\hline - Hyperthyroidism & $2(2)$ \\
\hline - Hypothyroidism & $19(19)$ \\
\hline \multicolumn{2}{|l|}{ Indication for procedure, n (\%) } \\
\hline - Iron deficiency anemia & $53(53)$ \\
\hline - Gastrointestinal bleeding & $14(14)$ \\
\hline - Crohn's disease & $12(12)$ \\
\hline - Abdominal pain/bloating & $12(12)$ \\
\hline - Celiac disease & $5(5)$ \\
\hline - Diarrhea & $4(4)$ \\
\hline \multicolumn{2}{|l|}{ Medications, n (\%) } \\
\hline - Beta-blockers & $25(25)$ \\
\hline - Thyroid medications & $17(17)$ \\
\hline - Calcium-channel blockers & $15(15)$ \\
\hline - Anticholinergics & $10(10)$ \\
\hline - Narcotics & $8(8)$ \\
\hline Adequate bowel preparation, n (\%) & $100(100)$ \\
\hline
\end{tabular}

The median capsule transit times were 22 minutes for gastric (interquartile range [IQR] 15-44; range 4-480), 176 minutes for the small bowel (IQR 116-250; range 11-407), and 227 minutes for the total bowel (IQR 157-280; range 33480).
- Table 2 Univariable logistic regression for video capsule endoscopy completion.

\begin{tabular}{|l|l|l|}
\hline Variable & OR $[95 \% \mathrm{Cl}]$ & P value \\
\hline Pedometer step count & & 0.17 \\
\hline Age & $1.20[0.92,1.55]$ & 0.65 \\
\hline Sex & $0.99[0.95,1.04]$ & 0.67 \\
\hline Body mass index & $1.44[0.27,7.83]$ & 0.02 \\
\hline Diabetes mellitus type 2 & $1.20[1.03,1.39]$ & 0.03 \\
\hline Inflammatory bowel disease & $0.17[0.03,0.82]$ & 0.62 \\
\hline Hypothyroidism & $0.56[0.10,3.13]$ & 0.51 \\
\hline Hyperthyroidism & N/A & 0.99 \\
\hline $\begin{array}{l}\text { Indication (iron deficiency } \\
\text { anemia vs. others) }\end{array}$ & $1.55[0.33,7.31]$ & 0.58 \\
\hline Findings (normal vs. others) & $7.61[0.88,65.73]$ & 0.07 \\
\hline Medications & & \\
\hline - Narcotics & $0.17[0.03,1.08]$ & 0.07 \\
\hline - Anticholinergics & N/A & 0.97 \\
\hline - Beta-blockers & $0.41[0.09,1.99]$ & 0.27 \\
\hline - Calcium-channel blockers & N/A & 0.96 \\
\hline - Thyroid medications & $0.48[0.09,2.71]$ & 0.41 \\
\hline OR, odds ratio; Cl, confidence interval; N/A, not applicable. & \\
\hline Pedometer step count includes 500 steps as 1 unit. & \\
\hline
\end{tabular}

The median step count was 2782 steps (IQR 1699-3964; range $130-11392)$. In univariable analysis, step count was not significantly associated with VCE completion (OR 1.20, 95 $\% \mathrm{Cl} 0.92,1.55)$. BMI and diabetes mellitus type 2 were significantly associated with completion, and narcotic medication use also met criteria for inclusion in the multivariable model ( $\triangleright$ Table 2). In multivariable analysis, step count was not significantly associated with VCE completion (OR 1.45, 95\% Cl 0.84, 2.49). Higher BMI remained significantly associated with increased odds of completion whereas diabetes mellitus type 2 and narcotic use were associated with lower odds ( $\triangleright$ Table 3 ).

Step count was significantly associated with a shorter total transit time in univariable analysis (HR 1.08, $95 \% \mathrm{Cl} 1.02,1.14$ ) ( $>$ Fig.1). In addition, BMI was associated with shorter total transit times, and diabetes mellitus type 2 was associated with longer total transit times ( $\triangleright$ Table 4 ). In the multivariable model, increased step count was associated with shorter total transit times (HR 1.09, $95 \% \mathrm{Cl} 1.03,1.16)$. Higher $\mathrm{BMI}$ also remained associated with shorter total transit times ( $\downarrow$ Table 5 ).

Step count was significantly associated with shorter gastric and small-bowel transit times in multivariable analysis. For every 500 steps taken, gastric transit completion hazard increased $5 \%$ (HR 1.05, $95 \% \mathrm{Cl} 1.00,1.11)$ and small-bowel transit completion hazard increased $7 \%$ (HR 1.07, $95 \% \mathrm{Cl} 1.01,1.14)$. 
- Table 3 Multivariable logistic regression for video capsule endoscopy completion.

\begin{tabular}{|c|c|c|}
\hline Variable & OR $[95 \% \mathrm{Cl}]$ & $P$ value \\
\hline Pedometer step count ${ }^{1}$ & $1.45[0.84,2.49]$ & 0.19 \\
\hline Body mass index & $1.87[1.18,2.97]$ & 0.01 \\
\hline Diabetes mellitus type 2 & $0.02[<0.001,0.78]$ & 0.04 \\
\hline Narcotics & $0.01[<0.001,0.33]$ & 0.01 \\
\hline
\end{tabular}

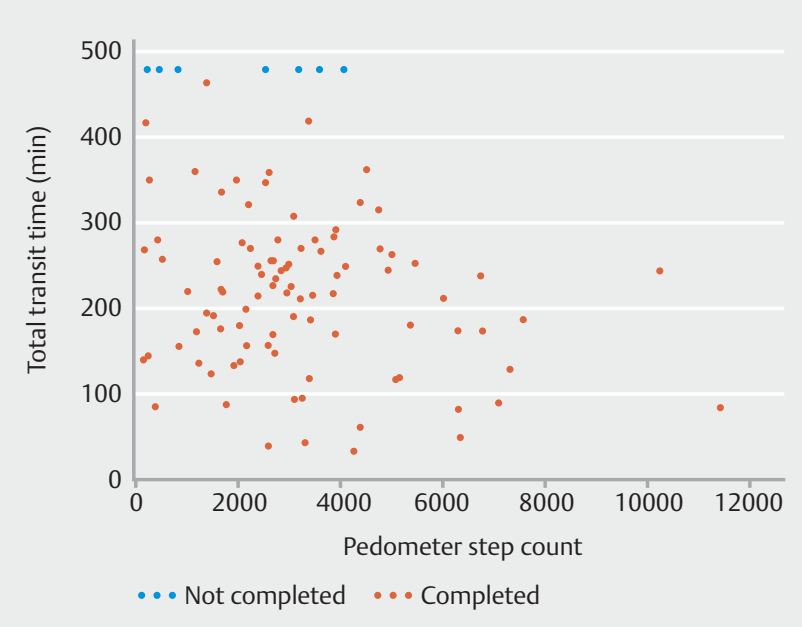

- Fig. 1 Scatterplot of total transit time by step count. Closed circles represent a completed video capsule endoscopy (VCE) study, and open circles represent incomplete VCE study.

\section{Discussion}

VCE is the preferred modality for direct assessment of the small-bowel mucosa but the device is limited by battery lifespan. Incomplete examinations can lead to missed pathology in the nonvisualized bowel and the need for repeated tests (with the associated increase in potential risks and healthcare costs). As such, methods to improve capsule completion without associated cost or side-effects would be high-yielding. Physical activity has previously been associated with accelerated small-bowel transit $[6,7]$. In our study, we prospectively investigated physical activity and VCE completion and transit times.

Step count during the procedure was not significantly associated with VCE completion in our cohort. However, there was a significant association between increasing step count and shorter total bowel transit time. We suspect the shorter bowel transit time did not translate into higher rates of VCE completion because of the high baseline completion rate of the study population. This suggests that increasing activity may be more beneficial in patient populations with prolonged bowel transit and a higher risk of incomplete studies, although this needs further investigation. Given the low-risk nature of increasing the
- Table4 Univariable Cox proportional hazards model for video capsule endoscopy total transit time.

\begin{tabular}{|l|l|l|}
\hline Variable & HR [95\%Cl] & P value \\
\hline Pedometer step count ${ }^{1}$ & $1.08[1.02,1.14]$ & 0.005 \\
\hline Age & $1.00[0.99,1.01]$ & 0.60 \\
\hline Sex & $0.90[0.59,1.38]$ & 0.63 \\
\hline Body mass index & $1.04[1.01,1.07]$ & 0.01 \\
\hline Diabetes mellitus type 2 & $0.54[0.32,0.93]$ & 0.03 \\
\hline Inflammatory bowel disease & $1.02[0.49,2.11]$ & 0.96 \\
\hline Hypothyroidism & $0.98[0.58,1.67]$ & 0.95 \\
\hline Hyperthyroidism & $1.24[0.30,5.08]$ & 0.76 \\
\hline Indication (iron deficiency & $1.19[0.79,1.79]$ & 0.41 \\
\hline anemia vs. others) & & 0.29 \\
\hline Findings (Normal vs. others) & $1.25[0.83,1.89]$ & \\
\hline Medications & & 0.46 \\
\hline - Narcotics & $0.73[0.32,1.68]$ & 0.53 \\
\hline - Anticholinergics & $1.24[0.64,2.39]$ & 0.42 \\
\hline - Beta-blockers & $0.82[0.51,1.32]$ & 0.22 \\
\hline - Calcium-channel blockers & $1.42[0.81,2.48]$ & $0.98[0.56,1.71]$ \\
\hline - Thyroid medications & 0.96 odds ratio; Cl, confidence interval. & \\
\hline OR & & 0.96 \\
\hline
\end{tabular}

- Table 5 Multivariable Cox proportional hazards model for video capsule endoscopy total transit time.

\begin{tabular}{|l|l|l|}
\hline Variable & HR [95\% $\mathbf{C l})$ & $\boldsymbol{P}$ value \\
\hline Pedometer step count & \\
\hline Body mass index & $1.09[1.03,1.16]$ & 0.003 \\
\hline Diabetes mellitus type 2 & $1.05[1.02,1.08]$ & 0.001 \\
\hline OR, odds ratio; $\mathrm{Cl}$, confidence interval. & $0.64[0.37,1.12]$ & 0.12 \\
\hline
\end{tabular}

amount of physical activity during the study, we do feel that ambulation should be recommended to patients during VCE.

Other clinical characteristics were significantly associated with VCE completion rate. Most notably, higher BMI was associated with increased video capsule completion and shorter total bowel transit times. This finding is supported by the gastrointestinal motility literature, which has linked underweight status with slower bowel transit times [14]. This is remarkable as $\mathrm{BMI}$ is not included as a clinical characteristic in most other series assessing completion rates and may be an uncontrolled factor affecting previously published results. To our knowledge, the series by Spada et al. assessing bowel transit times during VCE in patients with Celiac disease and the work by our group assessing opioid use and VCE completion, are the only studies to include BMI as a variable $[15,16]$. Neither study reported a significant correlation but both are limited by a retrospective 
study design. Until this is clarified, we recommend that BMI should be included in future studies as a variable of interest.

We found that the use of narcotic medication was associated with incomplete VCE. This is in contrast to our previously published experience and has been found to be of variable significance in other studies $[5,16,17]$. Diabetes mellitus type 2 was also found to be significantly associated with incomplete VCE. Although diabetes was initially linked to decreased completion rates by Triantafyllou et al., more recent reports have not shown a significant association $[3,17,18]$. These factors need to be considered when discussing the potential outcomes and risks of VCE with patients, but should not serve as a deterrent to the procedure.

The limitations of our study include the inability to directly correlate the timing of the physical activity with the location of the capsule owing to the modality of activity measurement. It should be noted that our ability to detect differences due to uncommon indications or VCE findings, such as active gastrointestinal bleeding, was limited by the sample size. In addition, the sample size probably also limited our ability to detect a difference in VCE completion given the high baseline completion rate of VCE performed on outpatients. Future studies should consider focusing on hospitalized patients as a target for interventions for VCE completion, given the lower completion rates in this patient population [3-5].

The strengths of our study include being the first study to use a validated tool to directly measure physical activity during the VCE study period. In addition, our prospective design allowed us to statistically control for multiple variables that affect capsule completion and bowel transit times. This overcomes a major limitation of previously published series assessing VCE and physical activity.

In conclusion, increased physical activity during outpatient VCE correlated with shorter bowel transit times but was not significantly associated with VCE completion. Given the lack of side-effects, we recommend that physical activity should be encouraged during VCE studies. In addition, BMI was a previously unreported clinical characteristic associated with VCE completion and should be included as a variable of interest in future studies assessing VCE completion.

\section{Acknowledgment}

We would like to thank Troy Thrash, Rebecca Ray, and Debra Graham for their dedication and vital contributions to this study. We also thank Alpha Omega Alpha for funding the study.

\section{Competing interests}

Marty M. Meyer - Speaker’s bureau Medtronic

\section{References}

[1] Gong F, Swain P, Mills T. Wireless endoscopy. Gastrointest Endosc 2000; 51: $725-729$

[2] Liao Z, Gao R, Xu C et al. Indications and detection, completion, and retention rates of small-bowel capsule endoscopy: a systematic review. Gastrointest Endosc 2010; 71: $280-286$

[3] Westerhof ], Weersma RK, Koornstra JJ. Risk factors for incomplete small-bowel capsule endoscopy. Gastrointest Endosc 2009; 69: 74 80

[4] Robinson CA, Jackson C, Condon D et al. Impact of inpatient status and gender on small-bowel capsule endoscopy findings. Gastrointest Endosc 2011; 74: 1061 - 1066

[5] Yazici C, Losurdo J, Brown MD et al. Inpatient capsule endoscopy leads to frequent incomplete small bowel examinations. World J Gastroenterol 2012; 18: 5051 - 5057

[6] Keeling WF, Martin B]. Gastrointestinal transit during mild exercise. J Appl Physiol 1987; 63: 978-981

[7] Dainese R, Serra J, Azpiroz F et al. Effects of physical activity on intestinal gas transit and evacuation in healthy subjects. Am J Med 2004; 116: $536-539$

[8] Shibuya T, Mori H, Takeda T et al. The relationship between physical activity level and completion rate of small bowel examination in patients undergoing capsule endoscopy. Intern Med 2012; 51: 997 1001

[9] Tudor-Locke C, Williams JE, Reis JP et al. Utility of pedometers for assessing physical activity: convergent validity. Sports Med 2002; 32: 795-808

[10] Bassett DR Jr. Validity and reliability issues in objective monitoring of physical activity. Res Q Exerc Sport 2000; 71: S30 - 36

[11] Schneider PL, Crouter SE, Lukajic O et al. Accuracy and reliability of 10 pedometers for measuring steps over a $400-\mathrm{m}$ walk. Med Sci Sports Exerc 2003; 35: 1779-1784

[12] Schneider PL, Crouter S, Bassett DR. Pedometer measures of free-living physical activity: comparison of 13 models. Med Sci Sports Exerc 2004; 36: $331-335$

[13] Shim KN, Jeon SR, Jang HJ et al. Quality indicators for small bowel capsule endoscopy. Clin Endosc 2017; 50: 148 - 160

[14] Sadik R, Stotzer PO, Simren M et al. Gastrointestinal transit abnormalities are frequently detected in patients with unexplained GI symptoms at a tertiary centre. Neurogastroenterol Motil 2008; 20: $197-$ 205

[15] Spada C, Riccioni ME, Urgesi R et al. Capsule endoscopy in celiac disease. World J Gastroenterol 2008; 14: 4146-4151

[16] Kleinman B, Stanich PP, Betkerur K et al. Opioid use is not associated with incomplete wireless capsule endoscopy for inpatient or outpatient procedures. Diagn Ther Endosc 2014; 2014: 651259

[17] Lee MM, Jacques A, Lam E et al. Factors associated with incomplete small bowel capsule endoscopy studies. World J Gastroenterol 2010; 16: $5329-5333$

[18] Triantafyllou K, Kalantzis C, Papadopoulos AA et al. Video-capsule endoscopy gastric and small bowel transit time and completeness of the examination in patients with diabetes mellitus. Dig Liver Dis 2007; 39: $575-580$ 